\title{
Giant Intracranial and Extracranial Meningioma of the Falx Cerebri: Surgical Management and Case Report
}

\author{
Alejandro Rojas-Marroquín ${ }^{1,2 *}$, Hugo Navarro Palencia ${ }^{3,4}$, Alejandro Curán ${ }^{5}$, Luis A. Ramírez ${ }^{6}$ \\ ${ }^{1}$ Department of Neurosurgery, Medilaser Clinic, Florencia, Colombia \\ ${ }^{2}$ Endovascular Neurosurgeon, Universidad Nacional Autónoma de México, México DF, México \\ ${ }^{3}$ Department of Maxillofacial Surgery, Medilaser Clinic, Florencia, Colombia \\ ${ }^{4}$ Oral and Maxillofacial Surgeon, Universidad Militar Nueva Granada, Bogotá, Colombia \\ ${ }^{5}$ Fellowship of Oral and Maxillofacial Surgery, Universidad Javeriana, Bogotá, Colombia \\ ${ }^{6}$ Universidad Cooperativa de Santa Marta, Santa Marta, Colombia \\ Email: alejorojasm@gmail.com
}

Received 23 May 2015; accepted 30 June 2015; published 3 July 2015

Copyright (C) 2015 by authors and Scientific Research Publishing Inc.

This work is licensed under the Creative Commons Attribution International License (CC BY).

http://creativecommons.org/licenses/by/4.0/

c) (i) Open Access

\begin{abstract}
Meningiomas are usually benign growths that rarely invade the substance of the brain, thus presenting the potential for curative surgery. Their sometimes difficult locations and propensity to recur if not completely excised represent formidable problems for the patient and the neurologic surgeon. This report describes a case of giant intracranial and extracranial meningioma of the falx cerebri. The tumor had displaced the anterior third of the superior sagittal sinusand a massive extracranial protrusion was eroding the calvarium, left supraorbital rim, frontal sinus and the left maxillary sinus. We present the surgical approach.
\end{abstract}

\section{Keywords}

Meningioma, Intracranial, Extracranial, Superior Sagittal Sinus, Surgical Approach

\section{Introduction}

Meningiomas are usually benign brain tumors characterized by progressive slow growth and presentation with subtle signs despite the frequently encountered large size. Meningiomas with predominantly extracranial extension are relatively rare. The incidence rate of meningiomas is about 2.6/100,000 populations, being more frequent in women than in men. The ratio of male to female incidence ranges from 1:1.4 to 1:2.8 [1]. 
There is no single symptom or sign that alone identifies which patients harbor an intracranial meningioma. Indeed some tumors are identified fortuitously in patients who have no symptoms or signs of intracranial disease. Other patients have a variety of presenting features, including headache, paresis, seizures, personality change/ confusion and visual impairment.

The most common sites of meningiomas are: Parasagittal/falcine (25\%), in the convexity (19\%), in the sphenoid ridge (17\%) and suprasellar (9\%). Meningiomas of the falx cerebri are less common (20\% - 50\% less) that parasagittal tumors, more commonly bilateral and rarely involve the superior sagittal sinus [2]. As in the case of parasagittal meningioma, extensive central debulking decreases the need for cerebral retraction. Excision of the falx allows complete removal, as well as access to the opposite side for removal of contralateral tumor extension.

In this paper we describe a case of a giant intracranial and extracranial meningioma of the falx cerebri and the surgical approach.

\section{Case Report}

Male patient, 67 years old, from the Amazon, with clinical symptoms of 2 years of evolution of painful swelling in the left frontal region, which grew exponentially to reach a diameter of $20 \mathrm{~cm}$. The patient presented headache of moderate intensity and left visual loss, three ulcers on the dome of the tumor was also observed.

Because of their low educational level, socio-cultural characteristics, and the remoteness of where he lives in relation to any urban center, proved unworkable the possibility that this patient received medical help earlier.

On admission to the emergency room, brain CT scan was performed, and 3D reconstruction of the skull, from which intra-extra cranial giant tumor was documented, with dural tear, erosion and invasion of the left supraorbital rim, frontal sinus and the left maxillary sinus (Figures 1(a)-(c)). Surgery was decided together with maxillofacial surgery.

Patient supine and in neutral position (Figure 2(a) \& Figure 2(b)), anesthetic induction and intubation is performed. A bicoronal skin incision was marked to gain wide exposure. A scalp flap was raised. The scalp was reflected to expose frontal bone and left supraorbital rim. A bifrontal craniotomy was performed. The tumor was firm and only moderately vascular. The large middle meningeal feeder was identified and coagulated early in the operation, over the lateral aspect of the capsule. Subsequently, the nasolabial fold incision was made, with left infraorbital extension to expose the extension of the tumor into the left maxillary sinus (Figure 2(c)). The tumor was excised, a clear plane between the mass and the surrounding brain became evident. Radical resection of the tumor was performed (Figure 3(a) \& Figure 3(b)). The tumor had displaced the anterior third of the superior sagittal sinus but not involved. The excised tumor mass weighed 400 gr. The patient lost 1 liter of blood

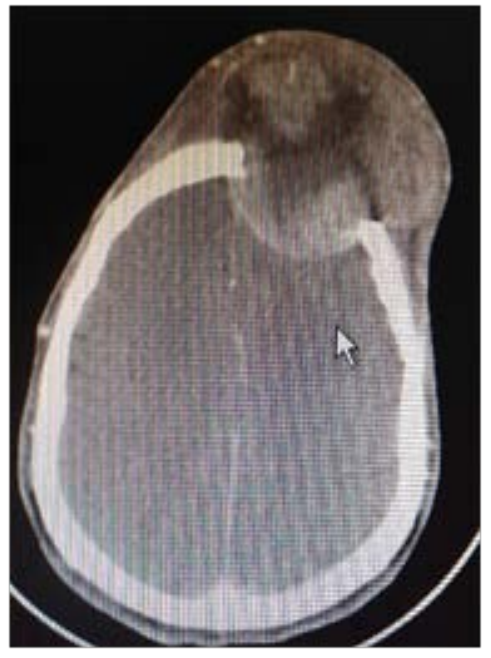

(a)

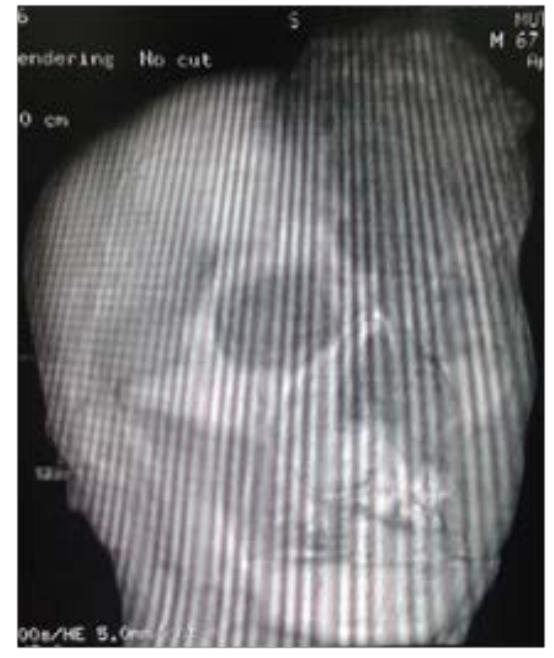

(b)

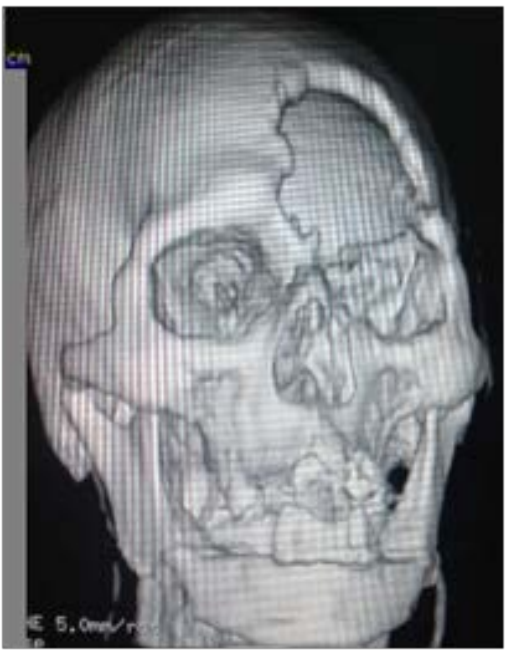

(c)

Figure 1. (a) Axial CT scan: giant intra- and extracranial meningioma. (b) and (c) 3D skull reconstruction: soft tissue and bone view. 


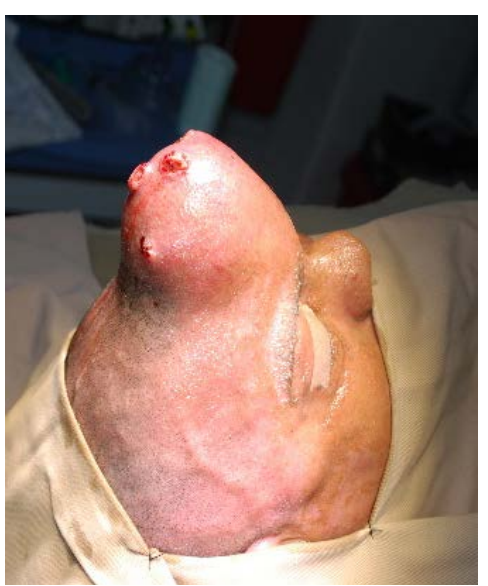

(a)

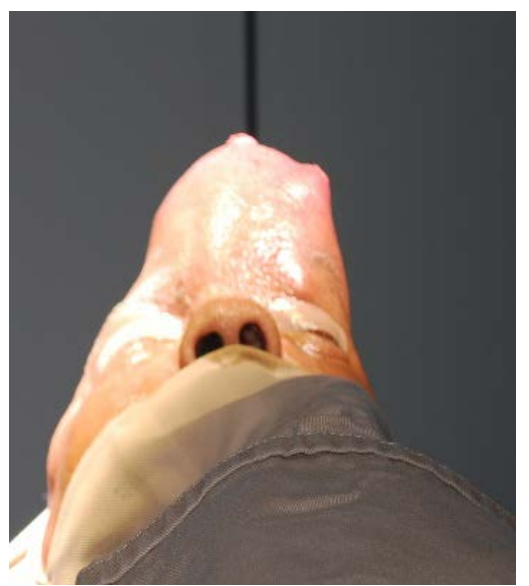

(b)

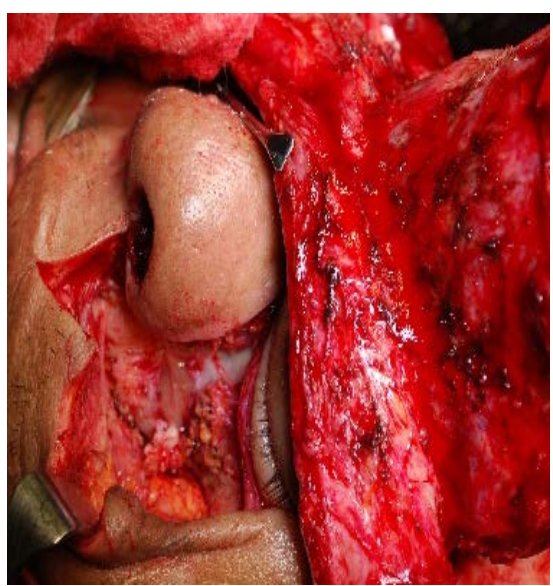

(c)

Figure 2. (a) and (b): AP and lateral view of the tumor. (c): Surgical approach: extracranial neoplastic protrusion extending to the left maxillary sinus.

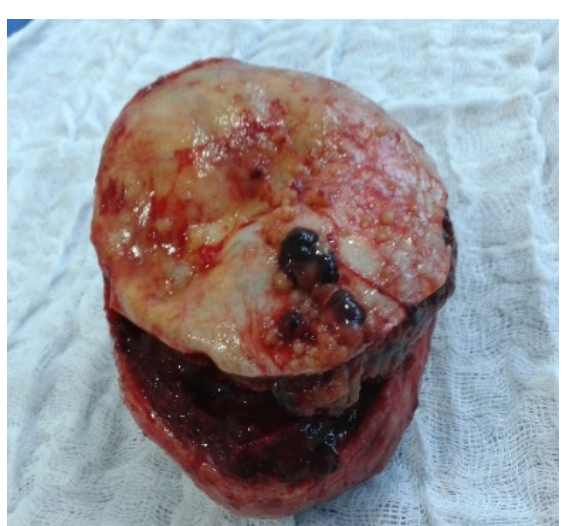

(a)

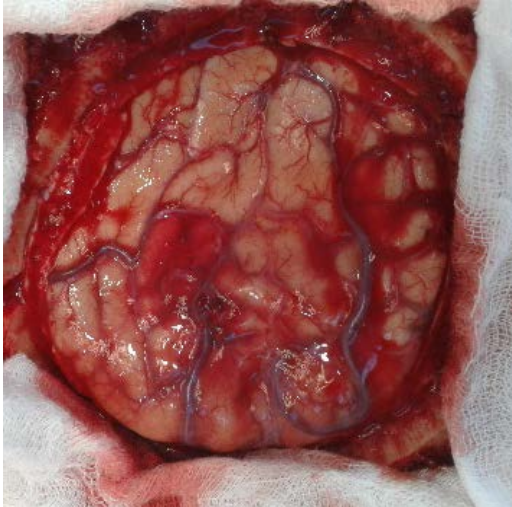

(b)

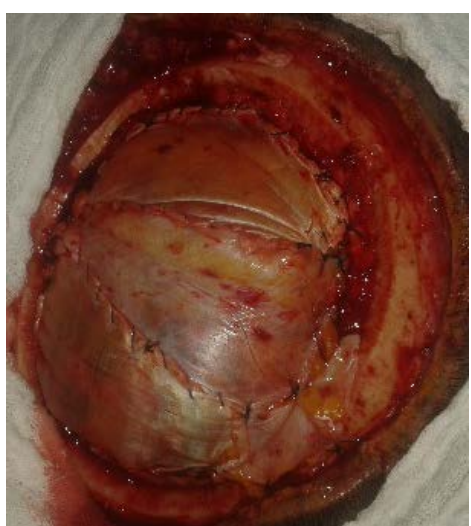

(c)

Figure 3. (a) and (b) Radical resection of the tumor. (c) Dural repair with fascia lata.

during the procedure of tumor resection.

Finally, at this point, careful dural repair was performed using pericranium, harvested from the adjoining region and is reinforced with fascia lata (Figure 3(c)). A myocutaneus skin flap reposition is performed (Figure 4(a) \& Figure 4(b)).

Histological examination confirmed the presence of a meningothelial meningioma (Figure 5(a)). There was no evidence of malignancy.

For its part, postoperative CT scan showed radical resection of the tumor (Figure 6(a)). There were no intraoperative or postoperative complications. The post operative recovery of the patient was uneventful.

\section{Discussion}

Meningiomas account for about 20\% of all intracranial tumours and, as slow-growing tumours that display benign behaviour, can escape notice. However, large hyperosteoting meningiomas and those occurring in extracranial locations are especially rare.

Cushing and Eisenhardt addressed some cases of giant meningioma described in the $18^{\text {th }}$ and $19^{\text {th }}$ centuries [3], but today as a result of modern diagnostic techniques such cases are rarely encountered.

In 1937 Davidoff removed an 835 gr tumour and two recent cases were described by Cech et al. and Djindjian and Raulo. In the case of Cech et al. the tumour was a parietooccipital meningioma, the tumor weighed $2600 \mathrm{gr}$ [4]. In the case reported by Djindjian and Raulo an 800 gr parietal meningioma was removed in a two-stage operation after embolization of the feeding arteries. 


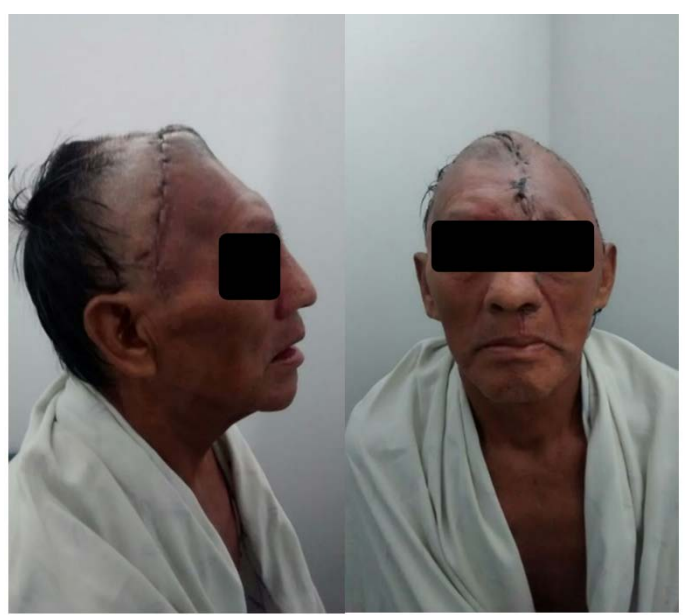

(a)

(b)

Figure 4. (a) and (b) Surgical wound.

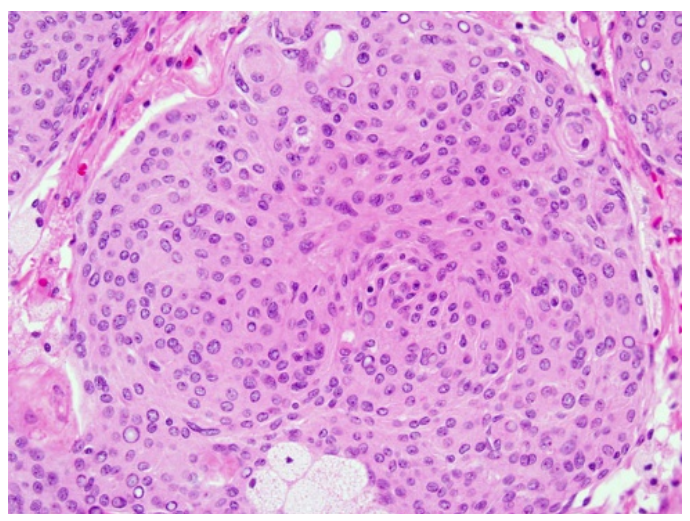

Figure 5. Photomicrograph of the Meningothelial meningioma. The nuclear size is rather uniform, oval in shape and without prominent nucleoli. Abundant intranuclear pseudo inclusions and intranuclear clear vacuoles are observed.

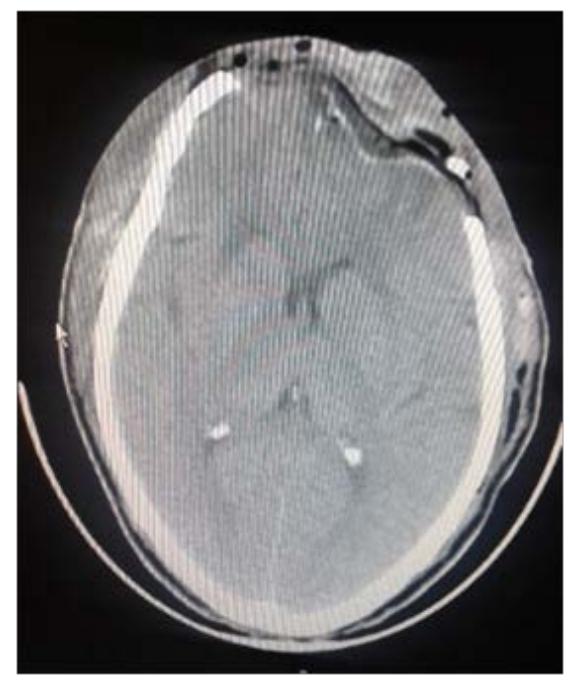

Figure 6. Brain CT scan: Absence of tumor. 
The symptoms are non-specific, usually associated with headache, paresis, seizures, personality change/confusion and visual impairment. The diagnosis is made through brain CT scan, ideally with axial and coronal view to define more clearly the intracranial component. On the other hand, MRI (magnetic resonance imaging) of the brain further defines the tumor and surrounding anatomical structures.

Percutaneous needle biopsy can establish the diagnosis. Nevertheless, in our case, we prefer not to do it and send the entire tumor to allow histological examination.

Surgical treatment of these giant tumors, as is known, presents various problems; among them, the most important are: massive intraoperative bleeding and ulceration surface generating a source of infection, and it was represented a common cause of death in the past. The recommendation, to avoid massive bleeding during tumor resection, is the preoperative embolization [5] [6].

In our case, digital subtraction angiography showed the moderately vascular tumor. The vascular supply was mainly from the external carotid circulation, predominantly through the middle meningeal artery. Endovascular embolization was not performed due to the moderate vascularity of tumor and the possibility of compromising scalp vascularity.

Another complication is, of course, the bleeding of venous sinuses [7]. In our case, the superior sagittal sinus was displaced by the tumor, but was not infiltrated or invaded.

An additional problem is the surgical reconstruction of the scalp, which, in the case at hand, a rotation of cutaneous and myocutaneous flap is made by completing the zetaplastia.

Complete surgical excision of meningiomas has been shown to offer the best long-term outcome compared with subtotal excision with or without radiotherapy. However, even with optimum surgical excision, recurrencerates of up to $20 \%$ can be expected over a 20 -year period [8] [9].

\section{References}

[1] Longstreth, W.T., Dennis, L.K., McGuire, V.M., et al. (1993) Epidemiology of Intracranial Meningioma. Cancer, 72, 639-648. http://dx.doi.org/10.1002/1097-0142(19930801)72:3<639::AID-CNCR2820720304>3.0.CO;2-P

[2] Walker, A.E., Robins, H. and Weinfeld, F.D. (1985) Epidemiology of the Brain Tumors: The National Survey of Intracranial Neoplasms. Neurology, 32, 219-226. http://dx.doi.org/10.1212/WNL.35.2.219

[3] Cushing, H. and Eisenhardt, L. (1938) Meningiomas: Their Classification, Regional Behaviour, Life History, and Surgical Ends Results. Charles C. Thomas, Springfield.

[4] Cech, D.A., Leavens, M.E. and Larson, D.L. (1982) Giant Intracranial and Extracranial Meningioma: Case Report and Review of the Literature. Neurosurgery, 11, 694-697. http://dx.doi.org/10.1227/00006123-198211000-00015

[5] Manelfe, C., Lasjaunias, P. and Ruscalleda, J. (1986) Preoperative Embolization of Intracranial Meningiomas. American Journal of Neuroradiology, 7, 963-972.

[6] Sindou, M.P. and Alaywan, M. (1998) Most Intracranial Meningiomas Are Not Cleavable Tumors: Anatomic-Surgical Evidence and Angiographic Predictability. Neurosurgery, 42, 476-480. http://dx.doi.org/10.1097/00006123-199803000-00007

[7] Bonnal, J. and Brotchi, J. (1978) Surgery of the Superior Sagittal Sinus in Parasagittal Meningiomas. Journal of Neurosurgery, 48, 938-945. http://dx.doi.org/10.3171/jns.1978.48.6.0935

[8] Rao, S.B., Dinakar, I. and Rao, K.S. (1971) Giant Intracranial Epidural Meningioma: Case Report. Journal of Neurosurgery, 35, 748-750. http://dx.doi.org/10.3171/jns.1971.35.6.0748

[9] Younis, G. and Sawaya, R. (1992) Intracranial Osteolytic Malignant Meningiomas Appearing as Extracranial Soft Tissue Masses. Neurosurgery, 30, 932-934. http://dx.doi.org/10.1227/00006123-199206000-00022 